\title{
Fatores associados ao tipo de parto em mulheres brasileiras: PNDS 2006
}

\author{
Factors associated with the type of delivery in Brazilian women: \\ 2006 NDHS
}

Fernanda de Oliveira Meller ${ }^{1}$

Antônio Augusto Schäfer ${ }^{1}$

${ }^{1}$ Universidade Federal de Pelotas. Rua Gomes Carneiro 01 Campus Porto, Centro. 96010-610 Pelotas

RS.fe_meller@hotmail.com
Abstract The scope of this paper was to analyze the prevalence of the types of delivery and associated factors in Brazilian women of childbearing age studied in the last National Demographics and Health Survey (NDHS). The study is a national domicile-based cross-sectional analysis, seeking to establish the characteristics of the female population of child-bearing age and of children under 5 years of age. The prevalence of the type of delivery was described according to the following exposition variables: Brazilian macroregions, location of residence, age, skin color, nutritional status, education, prenatal health unit and place of delivery. The statistical significance $(p<0,05)$ was evaluated using the chi-square test. The population studied consisted of 6.125 women. It was revealed that $42.9 \%$ of the women had a caesarean section. Significantly, the prevalence of caesarean section was smaller, irrespective of macro-regions, location of residence, age, skin color, nutritional status, prenatal health unit and place of delivery. The need for further investigation into the factors behind the increase in caesarean sections in Brazil is required in order to reverse this situation, since caesarean section can lead to a series of health complications for both mother and child.

Key words Brazilian women, Type of delivery, Risk factors
Resumo $O$ objetivo do trabalho foi analisar a prevalência dos tipos de parto e fatores associados em mulheres brasileiras em idade fértil estudadas na última Pesquisa Nacional de Demografia e Saúde (PNDS). O estudo compreende um recorte da PNDS, a qual se trata de um estudo transversal, de base domiciliar, de âmbito nacional, que teve como objetivo central caracterizar a população feminina em idade fértil e as crianças menores de cinco anos. Foram descritas as prevalências de tipo de parto segundo as seguintes variáveis de exposição: macrorregiões brasileiras, situação de domicílio, idade, cor da pele, estado nutricional, escolaridade, local do pré-natal e local do parto. A significância estatística $(p<0,05)$ foi avaliada pelo teste do qui-quadrado. A população estudada constituiu-se de 6.125 mulheres. Observou-se que 42,9\% das mulheres tiveram parto cesariano. A prevalência de parto cesariano foi significantemente menor, independente da macrorregião de moradia, situação de domicílio, idade, cor da pele, estado nutricional, local do pré-natal e do parto. Evidenciou-se a necessidade da continuidade na investigação dos fatores que promovem o aumento de partos cesarianos no Brasil, a fim de inverter os indices elevados, uma vez que pode levar a uma série de complicações tanto para as mães quanto para seus filhos.

Palavras-chave Mulheres brasileiras, Tipo de parto, Fatores de risco 
Introdução

A gestação representa período único e especial na vida da mulher, no qual a sensação de tornar-se mãe confunde-se muitas vezes com incertezas, medos e inseguranças. Esse fato aflora nas primigestas, especialmente no que se relaciona ao momento do parto. Em muitos casos, a escolha da via de parto motiva grande discussão clínica. Em geral, a gestante não participa dessa discussão, sendo, quando muito, informada sobre a decisão médica final. Não se leva em consideração sua aceitação ou não em relação à conduta a ser tomada, nem a associação entre a sua aceitação e os resultados perinatais obtidos ${ }^{1}$.

A cesariana é indicada com a intenção de salvar a vida da mãe e do filho em situação de alto risco, tais como: sofrimento fetal, apresentação pélvica, hemorragia antes do parto, doença hipertensiva específica da gravidez, gemelaridade, diabetes e cesariana de repetição ${ }^{2}$.

Nos últimos 30 anos, a taxa de cesariana tem aumentado, consideravelmente, em muitos países. Nos Estados Unidos, de acordo com Menacker et al. ${ }^{3}$, passou para $40 \%$ nos últimos 10 anos. Em Cuba, também foi evidenciado um aumento deste indicador nos últimos anos. ${ }^{4}$ Em 1997, o Brasil apresentou a maior taxa do mundo, chegando a $36,4 \%$, já em 2003, o percentual desse tipo de parto no Sistema Único de Saúde (SUS) diminuiu para $32,9 \% \%^{5,6}$.

A Organização Mundial da Saúde (OMS) considera que não há justificativa para um percentual superior a $15 \%$ em nenhuma região do mundo ${ }^{7}$. O Ministério da Saúde do Brasil, igualmente, considera que elevadas taxas de cesarianas são fatores determinantes da morbimortalidade materna e perinatal ${ }^{8}$. Além disso, esse tipo de parto associa-se com retardo na recuperação puerperal, maior tempo de internação, início tardio da amamentação, elevação de gastos para o sistema de saúde, entre outras complicações?.

As razões para esta alta prevalência parecem estar relacionadas a fatores que interferem na escolha da via do parto, bem como o estrato econômico, cultural e o acompanhamento profissional durante o pré-natal e o parto ${ }^{10}$.

Diante das elevadas taxas de partos cesarianos e de suas complicações tanto materna quanto perinatal, o presente estudo objetivou analisar a prevalência do tipo de parto e os fatores associados em mulheres brasileiras com idade entre 15 e 49 anos, estudadas na última Pesquisa Nacional de Demografia e Saúde (2006).

\section{Metodologia}

O presente estudo compreende um recorte da Pesquisa Nacional de Demografia e Saúde $(\mathrm{PNDS})^{11}$, inquérito de âmbito nacional, que teve como objetivo central caracterizar a população feminina em idade fértil e as crianças menores de cinco anos segundo fatores demográficos, socioeconômicos e culturais. Trata-se de um estudo transversal, de base domiciliar. Teve início no dia 3 de novembro de 2006 e foi concluído em 3 de maio de 2007.

O plano amostral da PNDS ${ }^{11}$ foi desenhado para fornecer estimativas representativas da população brasileira residente em domicílios particulares em setores comuns ou não especiais (inclusive favelas), selecionados em dez estratos amostrais que compõem uma combinação de todas as cinco grandes regiões geográficas brasileiras e as áreas urbanas e rurais. Foram priorizados setores urbanos das regiões metropolitanas nas nove capitais onde estão alocados os escritórios do Instituto Brasileiro de Opinião Pública e Estatística: São Paulo, Rio de Janeiro, Belo Horizonte, Brasília, Curitiba, Porto Alegre, Salvador, Recife e Fortaleza.

O estudo foi realizado por meio de uma amostra probabilística de domicílios obtida em dois estágios de seleção: as unidades primárias, que são os setores censitários, e as unidades secundárias, que são os domicílios particulares, ocupados ou não.

O total de setores em cada estrato foi obtido de forma a garantir um total de entrevistas que permitisse um número mínimo de coleta de sangue em crianças, segundo os percentuais de incidência de deficiência de vitamina A no sangue.

A amostra de setores foi obtida de forma independente em dez estratos, por amostragem aleatória simples por conglomerados.

Os domicílios foram selecionados dentro dos setores, selecionando-se aleatoriamente doze domicílios por setor para responderem ao questionário completo, os quais são denominados domicílios elegíveis por conterem pelo menos uma mulher de 15 a 49 anos de idade.

Ao final do trabalho de campo foram obtidas informações sobre 14.617 domicílios, dos quais 13.056 tinham pelo menos uma mulher elegível, totalizando 15.575 mulheres entrevistadas de 15-49 anos.

As informações da pesquisa foram coletadas em dois questionários que continham informações básicas sobre o domicílio e seus moradores habituais e informações detalhadas sobre o pú- 
blico-alvo, com relação a temas, como: reprodução, história de nascimentos, gravidezes, sexualidade, nutrição e medicamentos. Em todos os domicílios selecionados na amostra, o questionário, denominado Ficha do domicílio, foi aplicado a um dos moradores, preferencialmente ao responsável pelo domicílio e, na ausência deste, a um morador maior de quinze anos idade que soubesse fornecer informações sobre o domicílio e seus moradores. Em seguida, foi aplicado um questionário mais amplo, denominado Questionário da mulher, a todas as mulheres de 15 a 49 anos de idade identificadas como moradoras habituais do domicílio, o qual contemplou os seguintes módulos: características da entrevistada; reprodução; anticoncepção; acesso a medicamentos; gravidez e parto; alimentação e nutrição; conjugalidade e sexualidade; planejamento da fecundidade; características do cônjuge e trabalho da mulher; peso, altura e circunferência da cintura e coleta de sangue.

No caso de domicílio sorteado sem a presença de um elegível, isto é, de morador do sexo feminino com idade de 15 a 49 anos, foi aplicado somente a Ficha do domicílio até a pergunta sobre a educação do responsável pelo domicílio. Este domicílio foi substituído pelo domicílio elegível mais próximo na seqüência do arrolamento, para se obter o total de doze domicílios dentro de um mesmo setor.

Após a aplicação dos questionários, foram mensurados o peso e a altura de mulheres e crianças de acordo com as recomendações da $\mathrm{OMS}^{12}$. Essas medidas foram feitas duas vezes para cada pessoa, calculando-se a média aritmética de ambas. Essas medidas antropométricas foram coletadas por antropometristas treinados.

Os indivíduos com dezoito anos ou mais foram avaliados segundo critérios da $\mathrm{OMS}^{12}$, a qual recomenda que a avaliação antropométrica do estado nutricional desses indivíduos seja realizada a partir do Índice de Massa Corporal (IMC), obtido pela divisão do peso, expresso em kg, pela altura, expressa em metros, e elevada ao quadrado. Foram considerados com sobrepeso os que apresentaram valores iguais ou superiores a 25 $\mathrm{kg} / \mathrm{m}^{2}$ e com obesidade quando os valores de IMC eram iguais ou superiores a $30 \mathrm{~kg} / \mathrm{m}^{2}$. Os indivíduos entre 15 e 17 anos foram avaliados segundo Cole et al. ${ }^{13}$ que também recomenda a utilização do IMC, porém ajustado para a idade.

No presente estudo, para a variável de exposição "estado nutricional”, agruparam-se os IMC e a variável foi dicotomizada em presença ou ausência de excesso de peso, as quais foram defi- nidas como $\mathrm{IMC} \geq 25 \mathrm{Kg} / \mathrm{m}^{2}$ e $\mathrm{IMC} \leq 24,9 \mathrm{Kg} /$ $\mathrm{m}^{2}$, respectivamente.

O programa utilizado para a entrada de dados foi o CSPro (Census and Survey Processing System) software desenvolvido pelo Bureau do Censo Norte-Americano. O banco de dados da PNDS 2006 está publicamente disponibilizado no seguinte endereço: http://bvsms.saude.gov.br/bvs/ pnds/banco_dados.php. No presente estudo foi utilizado o programa SPSS 13.0 para a análise dos dados.

Para o presente estudo, foram descritas as prevalências de tipo de parto segundo as seguintes variáveis de exposição: macrorregiões brasileiras, situação de domicílio (urbana ou rural), idade, cor da pele, estado nutricional, escolaridade, local do pré-natal e local do parto. A significância estatística $(\mathrm{p}<0,05)$ foi avaliada pelo teste do qui-quadrado.

Para todas as pessoas entrevistadas com quinze anos ou mais, foi lido o termo de consentimento livre e esclarecido, com todas as informações sobre a pesquisa e assinado por aquelas que aceitaram participar. As mulheres solteiras menores de dezoito anos, além da assinatura desse termo, tiveram que obter a aprovação de um dos responsáveis em um termo de consentimento específico.

O projeto foi aprovado ad referendum pelo Conselho de Ética em Pesquisa do Centro de Referência e Treinamento DST/Aids da Secretaria de Estado da Saúde (SP) e, posteriormente, recebeu a aprovação do mesmo Comitê em 03/10/2005.

\section{Resultados e Discussão}

A partir dos 13.000 domicílios selecionados, 15.575 mulheres na faixa etária dos 15 a 49 anos participaram do estudo. Após selecionar a variável tipo de parto, a população estudada constituiu-se de 6.125 mulheres.

Na Tabela 1 verifica-se que a maioria $(39,4 \%)$ das mulheres residia na região Sudeste e na zona urbana do país $(79,1 \%)$. Observa-se que grande parte $(72,9 \%)$ encontrava-se na faixa etária entre 21-35 anos, era de cor não branca $(65,8 \%)$, não apresentava excesso de peso $(56,0 \%)$ e, cerca de $1 / 5$ não tinha escolaridade. Quanto ao local onde foi realizado o pré-natal e ao local do parto, a grande maioria utilizou o serviço do SUS, 75,5\% e $76,5 \%$, respectivamente. Verifica-se ainda na Tabela 1 que a maioria $(57,1 \%)$ das mulheres teve parto normal.

A Tabela 2 apresenta a prevalência de parto cesariano segundo as variáveis de exposição. Ob- 
serva-se que a prevalência deste foi maior entre as mulheres com idade entre 36 e 49 anos, naquelas de cor da pele branca, entre as que apresentavam excesso de peso, nas que utilizaram serviço particular para a consulta pré-natal e naquelas que realizaram o parto em hospital particular. Em relação às macrorregiões e à situação de domicílio, observou-se maior prevalência desse tipo de par-

Tabela 1. Descrição da amostra em termos de variáveis demográficas, antropométrica, socioeconômica e de saúde. Brasil, 2006. $(n=6.125)$

\begin{tabular}{|c|c|c|}
\hline Variáveis & $\mathbf{n}$ & $\%$ \\
\hline \multicolumn{3}{|l|}{ Macrorregião } \\
\hline Norte & 1354 & 10,6 \\
\hline Nordeste & 1189 & 29,2 \\
\hline Sudeste & 1156 & 39,4 \\
\hline Sul & 1167 & 13,0 \\
\hline Centro-Oeste & 1259 & 7,8 \\
\hline \multicolumn{3}{|l|}{ Situação de domicílio } \\
\hline Urbano & 3969 & 79,1 \\
\hline Rural & 2156 & 20,9 \\
\hline \multicolumn{3}{|l|}{ Idade (em anos) } \\
\hline $15-20$ & 710 & 13,4 \\
\hline $21-35$ & 4574 & 72,9 \\
\hline $36-49$ & 841 & 13,7 \\
\hline \multicolumn{3}{|l|}{ Cor da pele } \\
\hline Branca & 2072 & 34,2 \\
\hline Não branca & 3981 & 65,8 \\
\hline \multicolumn{3}{|l|}{ Estado nutricional } \\
\hline Sem excesso de peso & 3482 & 56,0 \\
\hline Com excesso de peso & 2569 & 44,0 \\
\hline \multicolumn{3}{|l|}{ Escolaridade } \\
\hline Sem escolaridade & 1308 & 24,3 \\
\hline 1 a 4 anos & 1597 & 28,2 \\
\hline 5 a 8 anos & 1381 & 22,8 \\
\hline 9 anos ou mais & 1552 & 24,7 \\
\hline \multicolumn{3}{|l|}{ Local do pré-natal } \\
\hline SUS & 4732 & 75,5 \\
\hline Convênio & 681 & 16,1 \\
\hline Particular & 500 & 8,3 \\
\hline Outro & 9 & 0,1 \\
\hline \multicolumn{3}{|l|}{ Local do parto } \\
\hline Casa & 231 & 1,8 \\
\hline Hospital pelo SUS & 4769 & 76,5 \\
\hline Hospital pelo convênio & 607 & 14,5 \\
\hline Centro de Saúde & 13 & 0,1 \\
\hline Hospital Particular & 491 & 6,9 \\
\hline Outro & 13 & 0,2 \\
\hline \multicolumn{3}{|l|}{ Tipo de parto } \\
\hline Normal & 3693 & 57,1 \\
\hline Cesáreo & 2432 & 42,9 \\
\hline
\end{tabular}

O maior número de valores ignorados $(n=287)$ foi para a variável "escolaridade". to entre as mulheres que residiam na região $\mathrm{Su}$ deste e na zona urbana do país.

Similarmente aos resultados encontrados no presente estudo, outros autores ${ }^{13,14}$ demonstraram que a idade materna tem sido diretamente relacionada à prevalência de cesarianas. Freitas et al. ${ }^{15}$ relataram que mulheres com idade superior a 30 anos têm maior probabilidade de realizarem parto cesariano do que mulheres com idade inferior a 20 anos. Neste mesmo sentido, Galtier-Dereure et al. ${ }^{16}$ e Seligman et al. ${ }^{17}$ também observaram que a faixa etária superior a 34 anos, bem como a obesidade, são os principais fatores de risco para realização de cesariana. Por outro lado, Pelloso et al. ${ }^{18}$ encontraram em sua pesquisa maiores taxas de parto cesariano em mulheres mais jovens. Estudo conduzido por Tedesco et al. ${ }^{1}$ observou que quanto maior a idade, maior a preferência pelo parto normal, revelando maior ponderação e reflexão sobre as conseqüências da via de parto, com o amadurecimento da mulher.

O levantamento de alguns autores mostrou que a taxa de cesarianas é, potencialmente, maior em mulheres de cor de pele branca quando comparadas às de pele negra ${ }^{19,20}$. Esses resultados foram semelhantes aos encontrados na presente investigação, os quais demonstraram que a chance de realizar parto cesariano foi maior em mulheres brancas quando comparadas às mulheres não brancas.

No presente estudo não foi evidenciada diferença significativa entre tipo de parto e escolaridade da mulher. Similarmente, estudos realizados por Silveira et al. ${ }^{21}$ e D'orsi et al. ${ }^{14}$ observaram que não houve associação estatisticamente significativa entre o nível educacional e o tipo de parto. Entretanto, diferentes estudos têm relatado maior ocorrência de cesariana entre mulheres com melhor nível educacional ${ }^{22,23}$.

Diversos estudos ${ }^{5,24,25}$ evidenciaram que a criação de um vínculo mensurado pela realização de consultas pré-natais tem sido fator de risco para a opção pela cesariana. Uma vez que esta associação não pode ser inteiramente explicada por um maior risco gestacional destas mulheres, tem sido sugerido que o tipo e a qualidade de informações e sugestões transmitidas pelos serviços de saúde durante o pré-natal possam influenciar o tipo de parto. No presente estudo, evidenciou-se maior prevalência de parto cesariano entre as mulheres que utilizaram o serviço particular para a realização da consulta pré-natal, indicando uma possível maior influência dos profissionais de saúde dos serviços privados para a realização desse tipo de parto. 
Tabela 2. Associação de variáveis demográficas, antropométrica, socioeconômica e de saúde com o tipo de parto. Brasil, 2006. $(n=6.125)$

\begin{tabular}{|c|c|c|c|c|c|}
\hline \multirow[b]{2}{*}{ Variáveis } & \multicolumn{2}{|c|}{ Parto normal } & \multicolumn{2}{|c|}{ Parto cesariano } & \multirow[b]{2}{*}{$\mathbf{p}$} \\
\hline & $\mathbf{n}$ & $\%$ & $\mathbf{n}$ & $\%$ & \\
\hline $\begin{array}{l}\text { Macrorregião } \\
\text { Norte } \\
\text { Nordeste } \\
\text { Sudeste } \\
\text { Sul } \\
\text { Centro-Oeste }\end{array}$ & $\begin{array}{l}943 \\
839 \\
618 \\
632 \\
661\end{array}$ & $\begin{array}{l}69,2 \\
69,1 \\
48,3 \\
50,6 \\
50,7\end{array}$ & $\begin{array}{l}411 \\
350 \\
538 \\
535 \\
598\end{array}$ & $\begin{array}{l}30,8 \\
30,9 \\
51,7 \\
49,4 \\
49,3\end{array}$ & $<0,01$ \\
\hline $\begin{array}{l}\text { Situação de domicílio } \\
\text { Urbano } \\
\text { Rural }\end{array}$ & $\begin{array}{l}2268 \\
1425\end{array}$ & $\begin{array}{l}54,8 \\
65,9\end{array}$ & $\begin{array}{r}1701 \\
731\end{array}$ & $\begin{array}{l}45,2 \\
34,1\end{array}$ & 0,01 \\
\hline $\begin{array}{l}\text { Idade (em anos) } \\
\qquad \begin{array}{l}15-20 \\
21-35 \\
36-49\end{array}\end{array}$ & $\begin{array}{r}497 \\
2765 \\
431\end{array}$ & $\begin{array}{l}71,8 \\
57,2 \\
41,9\end{array}$ & $\begin{array}{r}213 \\
1809 \\
410\end{array}$ & $\begin{array}{l}28,2 \\
42,8 \\
58,1\end{array}$ & $<0,01$ \\
\hline $\begin{array}{l}\text { Cor da pele } \\
\text { Branca } \\
\text { Não branca }\end{array}$ & $\begin{array}{l}1091 \\
2561\end{array}$ & $\begin{array}{l}51,4 \\
60,3\end{array}$ & $\begin{array}{r}981 \\
1420\end{array}$ & $\begin{array}{l}48,6 \\
39,7\end{array}$ & $<0,01$ \\
\hline $\begin{array}{l}\text { Estado nutricional } \\
\text { Sem excesso de peso } \\
\text { Com excesso de peso }\end{array}$ & $\begin{array}{l}2290 \\
1357\end{array}$ & $\begin{array}{l}63,9 \\
48,3\end{array}$ & $\begin{array}{l}1192 \\
1212\end{array}$ & $\begin{array}{l}36,1 \\
51,7\end{array}$ & $<0,01$ \\
\hline $\begin{array}{l}\text { Escolaridade } \\
\text { Sem escolaridade } \\
1 \text { a } 4 \text { anos } \\
5 \text { a } 8 \text { anos } \\
9 \text { anos ou mais }\end{array}$ & $\begin{array}{l}815 \\
955 \\
819 \\
947\end{array}$ & $\begin{array}{l}60,2 \\
57,6 \\
54,6 \\
57,1\end{array}$ & $\begin{array}{l}493 \\
642 \\
562 \\
605\end{array}$ & $\begin{array}{l}39,8 \\
42,4 \\
45,4 \\
42,9\end{array}$ & 0,55 \\
\hline $\begin{array}{l}\text { Local do pré-natal } \\
\text { SUS } \\
\text { Convênio } \\
\text { Particular } \\
\text { Outro }\end{array}$ & $\begin{array}{r}3221 \\
149 \\
131 \\
5\end{array}$ & $\begin{array}{l}67,0 \\
25,0 \\
23,2 \\
34,0\end{array}$ & $\begin{array}{r}1511 \\
532 \\
369 \\
4\end{array}$ & $\begin{array}{l}33,0 \\
75,0 \\
76,8 \\
66,0\end{array}$ & $<0,01$ \\
\hline $\begin{array}{l}\text { Local do parto } \\
\text { Casa } \\
\text { Hospital pelo SUS } \\
\text { Hospital pelo convênio } \\
\text { Centro de Saúde } \\
\text { Hospital Particular } \\
\text { Outro }\end{array}$ & $\begin{array}{r}231 \\
3251 \\
115 \\
10 \\
77 \\
9\end{array}$ & $\begin{array}{r}100,0 \\
66,2 \\
22,9 \\
68,2 \\
16,1 \\
56,9\end{array}$ & $\begin{array}{r}0 \\
1518 \\
492 \\
3 \\
414 \\
4\end{array}$ & $\begin{array}{r}0 \\
33,8 \\
77,1 \\
31,8 \\
83,9 \\
43,1\end{array}$ & $<0,01$ \\
\hline
\end{tabular}

Observou-se maior prevalência de parto cesariano entre as mulheres que realizaram o parto em hospital particular $(83,9 \%)$. Esse resultado também foi evidenciado em estudos realizados por Potter et al. ${ }^{26}$, Mandarino et al. ${ }^{9}$ e Mendoza-Sassi et al. ${ }^{27}$, os quais encontraram mais do que o dobro de parto cesariana na rede privada quando comparada à rede pública.

Comparativamente às macrorregiões, a região Sudeste foi a que apresentou maior prevalência de parto cesariano $(51,7 \%)$, em contrapartida, as menores taxas foram encontradas nas regiões Norte $(30,8 \%)$ e Nordeste $(30,9 \%)$. Altas taxas de cesarianas foram evidenciadas na PNDS-199628, as quais foram associadas às macrorregiões brasileiras, sendo a região Centro-Oeste a que apresentou o maior percentual $(49,0 \%)$ e as regiões Nordeste $(20,0 \%)$ e Norte $(25,0 \%)$, os menores.

Com base nos resultados obtidos, é possível concluir que, embora tenha-se observado uma elevada prevalência de parto cesariano na população estudada, encontrou-se uma menor quan- 
do comparado ao parto normal, independentemente da macrorregião de moradia, situação de domicílio, idade, cor da pele, estado nutricional, local do pré-natal e local do parto.

Evidencia-se a necessidade da continuidade na investigação dos fatores que promovem o aumento de partos cesarianos no Brasil, uma vez que podem ocasionar uma série de complicações tanto para as mães quanto para seus filhos. Além disso, os índices encontram-se muito acima do recomendado pela $\mathrm{OMS}^{7}$, demonstrando que políticas públicas dirigidas à redução das taxas de cesarianas no país são urgentes e necessárias.

\section{Colaboradores}

FO Meller e AA Schäfer participaram igualmente de todas as etapas de elaboração do artigo.

\section{Referências}

1. Tedesco RP, Maia Filho NL, Mathias L, Benez AL, Castro VCL, Bourroul GM, Reis FI. Fatores determinantes para as expectativas de primigestas acerca da via de parto. Rev Bras Ginecol Obstet 2004; 26(10):791-798.

2. Rocco R, Leite HV, Vasconcelos M, Cabral ACV. Morbidade associada à cesariana eletiva em portadores de HIV. Rev Bras Ginecol Obstet 2003; 25(5):323-328.

3. Menacker F, Declercq E, Macdorman MF. Cesarean delivery: background, trends, and epidemiology. Semin Perinatol 2006; 30(5):235-241.

4. Falcón VE, Sánchez MI, La Rúa A, González FY. Índice de cesárea primitiva: un problema resuelto? In: X Congreso Nacional de Obstetricia y Ginecología; 1997; La Habana.

5. Belizán JM, Althabe F, Barros FC, Alexander S. Rates and implications of caesarean sections in Latin America: ecological study. BMJ 1999; 319:1397-1400.

6. Kilsztajn S, Carmo MS, Machado LCJ, Lopes ES, Lima LZ. Caesarean sections and maternal mortality in São Paulo. Eur J Obstet Gynecol Reprod Biol 2007; 132(1):64-69.

7. World Health Organization (WHO). Appropriate technology for birth. Lancet 1985; 2(8452):436-437.

8. Brasil. Ministério da Saúde. Parto, aborto e puerpério, assistência humanizada à mulher. Brasília: Secretaria de Políticas de Saúde, Ministério da Saúde; 2001.

9. Mandarino NR, Chein MB, Monteiro Junior FC, Brito LM, Lamy ZC, Nina VJ, et al. Aspects related to choice of type of delivery: a comparative study of two maternity hospitals in Sao Luis, State of Maranhao, Brazil. Cad Saude Publica 2009; 25(7): 1587-1596.

10. Barbosa GP, Giffin K, Ângulo-Tuesta A, Gama AS, Chor D, D'orsi E, dos Reis AC. Parto cesárea: quem o deseja? Em quais circunstâncias? Cad Saude Publica 2003; 19(6):1611-1620. 
11. Brasil. Ministério da Saúde (MS). Relatório Final da Pesquisa Nacional de Demografia e Saúde. Brasília: Pesquisa Nacional de Demografia e Saúde da Criança e da Mulher; 2006. [acessado 2010 abr 29]. Disponível em: http://www.saude.gov.br/pnds2006

12. World Health Organization (WHO). Physical Status: the Use and Interpretation of Anthropometry. Report of a WHO Expert Committee. WHO Technical Report Series 854. Geneva: WHO; 1995.

13. Cole T, Bellizzi M, Flegal K, Dietz W. Establishing standard definition for child overweight and obesity worldwide: international survey. Br Med J 2000; 320:1-6.

14. D’orsi E, Chor D, Giffin K, Ângulo-Tuesta A, Barbosa GP, Gama AS, Reis AC. Factors associated with cesarean sections in a public hospital in Rio de Janeiro, Brazil. Cad Saude Publica 2006; 22(10): 2067-2078.

15. Freitas PF, Sakae TM, Jacomino MEMLP. Medical and non-medical factors associated with cesarean section rates in a university hospital in southern Brazil. Cad Saude Publica 2008; 24(5):1051-1061.

16. Galtier-Dereure F, Boegner C, Bringer J. Obesity and pregnancy: complications and cost. Am J Clin Nutr 2000; 71(Supl. 5):1242-1248.

17. Seligman LC, Duncan BB, Branchtein L, Gaio DSM, Mengue SS, Schmidt MI. Obesity and gestational weight gain: cesarean delivery and labor complications. Rev Saude Publica 2006; 40(3):457-465.

18. Pelloso SM, Panont KT, Souza KMP. Opção ou imposição! Motivos da escolha da cesárea. Arq Cien Saude Unipar 2000; 4(1):3-8.

19. Kabir AA, Pridijian G, Steinmann WC, Herrera EA, Khan MM. Racial differences in cesareans: an analysis of U.S. 2001. Obst Gynecol 2005; 105:710-718.

20. Linton A, Peterson MR, Williams TV. Effects of maternal characteristics on cesarean delivery rates among U.S. Department of Defense healthcare beneficiaries 1996-2002. Birth 2004; 31(1):3-11.

21. Silveira DS, Santos IS. Factors associated with cesarean sections among low-income women in Pelotas, Rio Grande do Sul, Brazil. Cad Saude Publica 2004; 20(Supl. 2):231-241.
22. Althabe F, Belizan JM. Caesarean section: the paradox. Lancet 2006; 368(9546):1472-1473.

23. Freitas PF, Drachler Mde L, Leite JC, Grassi PR. Social inequalities in cesarean section rates in primiparae, Southern Brazil. Rev Saude Publica 2005; 39(5):761-767.

24. Althabe F, Belizán JM, Villar J, Alexander S, Bergel E, Ramos S, Romero M, Donner A, Lindmark G, Langer A, Farnot U, Cecatti JG, Carroli G, Kestler E; Latin American Caesarean Section Study Group. Mandatory second opinion to reduce rates of unnecessary caesarean sections in Latin America: a cluster randomised controlled trial. Lancet 2004; 363(9425):1934-1940.

25. Barros AJD, Hirakata VN. Alternatives for logistic regression in cross-sectional studies: an empirical comparison of models that directly estimate the prevalence ratio. BMC Med Res Methodol 2003; 3:21-33.

26. Potter JE, Berquo E, Perpetuo IH, Leal OF, Hopkins K, Souza MR, Formiga MC. Unwanted caesarean sections among public and private patients in Brazil: prospective study. BMJ 2001; 323(7322):1155-1158.

27. Mendoza-Sassi RA, Cesar JA, Silva PR, Denardin G, Rodrigues MM. Risk factors for cesarean section by category of health service. Rev Saude Publica 44(1):80-89.

28. Sociedade Civil Bem-Estar Familiar do Brasil (BEMFAM). Pesquisa Nacional de Demografia e Saúde, 1996. Rio de Janeiro: Sociedade Civil Bem-Estar Familiar; 1996.

Artigo apresentado em 18/10/2010

Aprovado em 10/02/2011

Versão final apresentada em 20/02/2011 